\title{
Framework for nurse managers to evaluate the imple- mentation of a workplace wellness programme
}

\author{
Lizeth Roets ${ }^{1}$, Helena Basson ${ }^{2}$ \\ 1. Department of Health Studies, University of South Africa, South Africa. 2. School of Nursing, University of the Free State,
} South Africa

Correspondence: Lizeth Roets. Address: Department of Health Studies, School of Social Sciences, Theo van Wyk building, 7-183, Muckleneuk Campus, Unisa, South Africa 0003. Email: roetsl@unisa.ac.za.

Received: January 24, 2013

DOI : $10.5430 /$ jnep.v3n7p140
Accepted: March 25, $2013 \quad$ Online Published: June 7, 2013

URL: http://dx.doi.org/10.5430/jnep.v3n7p140

\section{Abstract}

Background: Management of HIV/AIDS in the healthcare workplace poses a challenge to nurse managers, as many nurses are affected by HIV. A workplace wellness programme (WWP) is a strategy that enhances organisational health and wellbeing among nurses infected and/or affected by HIV/AIDS. However to remain effective and sustainable, workplace programmes within the South African context must be evaluated.

Methods: A quantitative descriptive design with questionnaires was utilised. The data gathered, as well as the logic model, was used to develop a framework to enable nurse managers to evaluate the implementation of a workplace wellness programme.

Results: Respondents indicated what should be included and evaluated when implementing a WWP to ensure that such a programme is sustainable. The theory of change logic model was then utilised to develop the framework for evaluation.

Conclusion: Nurses indicated that the management of HIV/AIDS in their workplace must be reconsidered, and the focus should be on promotional interventions and services to enhance health and wellness. A framework for evaluating the success and sustainability of a programme was proposed and should be available to enhance the effectiveness of its implementation.

\section{Key words}

Evaluation framework, Workplace wellness programme, HIV/AIDS, Nurse, Theory of change logic model

\section{Introduction and background}

Promoting the health and wellbeing of nurses in the workplace is an obligation imposed upon all nurse healthcare managers in the health industry ${ }^{[1]}$ within countries where the HIV and AIDS figures are high. The World Health Organization (WHO) endorsed the importance of improving the health and wellbeing of all employees in the workplace ${ }^{\text {[2] }}$ by instituting the WHO's Global Healthy Work Approach in 1997, thus including the wellness of nurses infected with and/or affected by HIV/AIDS.

The effective management of the effect of human immunodeficiency virus and acquired immune deficiency syndrome (HIV/AIDS) in the healthcare workplace urgently required an integrated strategy to deal with the impact of HIV/AIDS on 
the workplace ${ }^{[3]}$. The strategy should address the health and the wellbeing of HIV/AIDS affected and/or infected nurses in the workplace. The establishment of an effective and sustainable workplace wellness programme (WWP) for nurses relies on appropriate evaluation strategies for implementation by nurse managers to underpin their co-responsibility for and commitment to promoting the health and wellbeing of all nurses.

An estimated 34 million people throughout the world were living with HIV at the end of 2010, while another 2.7 million people were newly infected. More than 1.8 million people died from AIDS, contributing to the fact that 16.6 million children were orphaned ${ }^{[4]}$.

These statistics are an indication that the healthcare industry and healthcare practitioners, in particular nurses, are affected by the impact of HIV/AIDS. Large numbers of sick and HIV-infected or AIDS patients need to be cared for; this contributes to additional responsibilities to nurses, low staff morale and fear of infection. Death rates among colleagues, patients, family and friends are high ${ }^{[4]}$. In the workplace, nurses must contend with a greater need for sick leave, higher absenteeism and the loss of highly qualified staff, all of which increase the workload of the remaining staff. HIV-infected nurses fear the loss of their jobs, discrimination and stigmatization ${ }^{[5-8]}$. These statistics support the need for a wellness programme in the workplace.

A literature review was carried out to determine and describe the components to be included and evaluated in a WWP for nurses who contend with HIV/AIDS in the workplace daily. Concepts such as health, wellness, health promotion and wellness programmes were searched through Google, Google Scholar, Pub Med as well as policy documents publicly available. The literature obtained was, studied and summarised. The literature review attempted to identify the interrelating and interacting factors between the nurse practitioner and his or her employer, usually represented by a nurse healthcare manager.

\section{Literature review}

The WWP must not only focus on rehabilitation and social assistance, but must provide guidelines for best practices to enable nurse healthcare managers to assist nurses with their wellbeing. The WWP should contribute to the enhancement of organisational wellbeing and improved attendance patterns, productivity and profitability ${ }^{[9]}$. It should attempt to move the focus to signs of health, indicating that the key to wellness is self-responsibility and the establishment of a positive approach ${ }^{[10]}$.

However, the value of a WWP can be questionable if effective evaluation strategies are not applied, to justify continuation or measure effective implementation. An evaluation strategy can help nurse managers to promote a supportive environment where the quality of life and morbidity, as well as mortality among HIV-infected or affected nurses, can be focused on and assessed.

Evaluation strategies should be developed to enable an organisation or nurse healthcare managers to measure a programme's progress against the stated goals and objectives of a company, institution or unit relating to HIV/AIDS. Evaluation results will support informed decisions on the effectiveness and appropriateness of a WWP and the HIV/AIDS activities in a workplace ${ }^{[6,11,12]}$.

The approach adopted by managers, in particular nurse healthcare managers governing a healthcare organisation, hospital, healthcare centre or unit was of the utmost importance in better comprehending, assessing and responding to the impact of HIV/AIDS in the healthcare sector ${ }^{[13]}$. Managerial factors such as sustaining efforts to create a supportive environment for optimal health and wellbeing had to be recognised ${ }^{[13]}$. Furthermore, internal factors such as organisational structure, policies on HIV/AIDS and organisational culture determine the feasibility and sustainability of a workplace intervention such as a WWP ${ }^{[3]}$. External factors such as national and international legislation, statements and policies must be taken into consideration ${ }^{[6]}$ when managing HIV/AIDS in a workplace. 
The rationale for an evaluation framework was examined as well. It was found that an evaluation framework could help in organising ideas, reaching conclusions and understanding events captured in a WWP ${ }^{[14,15]}$. However, explaining how a programme could successfully achieve its desired goals or objectives relied on the logic within such a framework. The logic is important, because it describes the intended theory of change supporting the framework ${ }^{[16]}$. The theory of change logic model ${ }^{[17]}$ was utilised as a model to underpin the suggested evaluation framework for a WWP. It supplied the theoretical guide for the development of the evaluation framework and gave meaning to the research findings.

The evaluation framework for a WWP should focus on promoting purposeful activities in support of the specific health goal of increasing the level of wellbeing and health of nurses. Furthermore, it can provide selective proceedings in the workplace to motivate and promote, empower and enhance, achieve and maintain the optimal health and wellbeing of the nurse practitioner infected with and/or affected by HIV/AIDS.

\section{Aim}

The aim of the study was to develop a proposed evaluation framework to assist nurse healthcare managers when evaluating the implementation of a WWP, intended to enhance the wellness of nurses infected with and/or affected by HIV/AIDS.

\section{Methodology}

\subsection{Design}

A quantitative descriptive research design was utilised to enable the researcher to obtain, identify and describe the information needed to develop a framework for evaluating a WWP.

\subsection{Population}

The population consisted of practising nurses registered with the South African Nursing Council. The accessible population was the 250 registered nurses employed at a private hospital in Bloemfontein, the capital city of the Free State province in South Africa.

\subsection{Sampling}

All 232 registered nurses working at the private hospital and who were available during the time of data gathering were asked to participate in the study on a voluntary basis. All were directly or indirectly affected by HIV/AIDS in the workplace ${ }^{[18]}$ owing to the nature of their nursing function in caring for sick patients, family, friends, colleagues or those affected by HIV and AIDS

\subsection{Measurement strategy}

After a thorough literature review was conducted, a structured questionnaire was developed. The questionnaire consisted of four sections, covering biographical information, nurses' opinions regarding the need for a wellness programme, the interventions and items to be included in the programme as well as personal information pertaining to HIV and how the nurses might be affected. The questionnaire included closed-ended questions, one open-ended question and some questions requiring the application of the Likert scale. The response choices addressed agreement options such as not at all important, not so important, fairly important and very important to encourage declarative statements from the respondents. The opinions or attitudes elicited from the nurses provided valuable information about components to be included in or managed through the evaluation framework ${ }^{[6,12]}$.

\subsection{Ethical procedure}

The questionnaire was submitted to a validation committee and a panel of experts in instrument development, research methodology and HIV/AIDS at the School of Nursing, at the university in Bloemfontein. This was done in collaboration 
with a study supervisor. Thereafter the ethicality of the measurement instrument was confirmed by the Ethics Committee of the Faculty of Health Sciences at the university, which granted final approval for the study.

In all, $141(n=141)$ questionnaires were anonymously and voluntarily completed, with due regard for confidentiality, integrity and honesty.

\subsection{Pilot study}

A pilot study with 8 respondents, was conducted at the sister hospital of the designated hospital, 5 kilometers away. No changes were made to the questionnaire that was completed in English. The respondents experienced the questions clear, concise and easy to answer and indicated that it took 15 minutes to complete. Respondents in the pilot study, as well as the data yielded, were not included in the main study.

\subsection{Data collection}

Following approval from the Ethics Committee of the Faculty of Health Sciences at the university and the management of the hospital group involved, as well as written permission from the respondents, the data was collected.

A questionnaire bundle consisting of a pre-packed sealable envelope containing a covering letter and an anonymous number-coded questionnaire with clear instructions for completion ${ }^{[19]}$ was distributed to each participant. A $61 \%$ (61\%; n $=141)$ response rate was obtained $(\mathrm{N}=232)$, which reinforced the significance of the study ${ }^{[18]}$.

\subsection{Data analysis}

The crude data was entered into a computer and numerically coded and organised by a biostatistician from the Department of Biostatistics at the university. Descriptive statistics, namely medians and percentiles for continuous data and frequencies and percentages for categorical data, were calculated, by utilizing the statistical Package for Social Sciences (SPSS 19.0). The relevance and inference between the literature review and the data obtained from the respondents were then captured and explained.

\section{Results}

\subsection{I nterpretation and application}

The respondents were asked why they considered a WWP important and what should be included, the data gathered as well as the theory of change logic model ${ }^{[16,17]}$ were then utilised to explain, develop and illustrate the framework for evaluation. What was important from the point of view of the nurse manager was to compile the framework to enable them to possibly evaluate the success and sustainability of such a programme. The framework can be used to evaluate whether a WWP succeeds in achieving its expected outcomes and to identify possible improvements in the form of alterations or amendments.

\subsection{Biographical data}

The respondents were predominantly female registered nurses representative of the sexually and economically active female adult population between the ages of 19 and 59 years. The majority of respondents were married and had to support their families financially.

\section{Response of nurses to HIV/ AI DS in the workplace}

The majority of respondents declared that they had been tested for HIV and diagnosed HIV negative; however 68\% reported that they were afraid of being infected with the disease. They wanted to stay HIV negative and were of the opinion that HIV/AIDS posed a serious threat to nurses in the workplace. The majority (69\%) of respondents indicated that 
although they experienced good health and wellbeing, symptoms of depression, tension or stress, and flu or the common cold were frequently experienced.

\section{HI V/ AI DS management and the workplace}

Of the respondents, $72 \%$ indicated that they considered an operational WWP very important to nurses. Another $20 \%$ felt a WWP to be important; thus the large majority of nurses (92\%) indicated the need for such a programme. They were aware of an existing workplace HIV/AIDS policy (75\%), workplace HIV/AIDS prevention and awareness programme (65\%), and an HIV/AIDS managerial training programme (57\%).

Of the respondents, 51\% were of the opinion that an HIV/AIDS task team was not adequately promoted, 55\% felt that voluntary confidential counselling and testing (VCCT) facilities were not adequately promoted and $48 \%$ considered that a peer educational programme was not adequately promoted, thus the successful implementation of a WWP should be evaluated.

Seventy two percent (72\%) felt that nurses should be allowed to be involved with the development of a workplace HIV/AIDS strategy and policy applicable to nurses. The proposal was made that the physical and psychosocial, as well as economic and occupational consequences of HIV/AIDS should be made explicit in a WWP, and that this should also include educative material and information for nurses. Whether these aspects are addressed in a WWP should be evaluated.

Seventy six percent (76\%) of respondents felt it to be the duty of a nurse healthcare manager to develop and co-ordinate employee wellness as a means of promoting and enhancing positive health, positive daily living principles and wellbeing among nurses. Nurse managers should therefore also be responsible for the evaluation of such a programme if they want to measure its success.

Seventy Six percent (76\%) of nurses expressed the need for information about managing stress, $72 \%$ said that they needed working hours that allowed enough resting periods, $72 \%$ stated that they needed to stay employed, and $71 \%$ expressed the need for protection initiatives against life-threatening diseases such as HIV/AIDS. 77\% of the respondents recommended that an occupational health nurse practitioner, skilled in HIV/AIDS issues, should always be available in the workplace. $77 \%$ stated that peer and counsellor education should be included in a WWP, and 67\% recommended the inclusion of an operational HIV/AIDS workplace task group in a WWP.

The nurses indicated that they would like the management of HIV/AIDS in their workplace to be reconsidered, and that the focus should be on promotional interventions and services to enhance health and wellness. A framework for evaluating the success and sustainability of such a programme should be available to nurse managers to enhance the effectiveness of its implementation. The evaluation framework should focus on:

- Assessing whether the WWP included actions to create an awareness of health and occupational topics that posed a hazard in the workplace and had an effect on nurses' wellbeing;

- Assessing whether the WWP contained effective health education, information and behaviour modification strategies to promote lifestyle changes and optimal health and wellbeing of nurses affected by or infected with HIV/AIDS;

- Assessing whether the WWP assisted in creating a supportive work environment that encouraged nurses to achieve and maintain positive health and wellness.

The literature review conducted as well as the research data obtained indicated the advisability of an integrated managerial strategy for the effective management of HIV/AIDS in the workplace. Thus, in the development of the wellness program and the evaluation thereof, all affected should be involved. It identified the objectives and components necessary for the 
introduction of a WWP and, what was important, the evaluation of this programme. An evaluation framework is necessary to help nurse managers measure whether the outcomes of the WWP are met.

\subsection{Evaluation framework}

An evaluation framework is a theoretical guide that helps people organise ideas, reach conclusions and understand events through the abstract and valid clarification and careful structuring of the knowledge acquired ${ }^{[14]}$. This view was obtained from the literature study and the opinions of nurses by means of the structured questionnaire described. The WWP that was developed incorporated theory, and this was tested by means of the evaluation framework ${ }^{[17]}$. The theory of change, in other words, how to bring about change in the workplace, has an influence on programme development, and therefore the theory of change logic model underpinned the development of the framework for evaluating the WWP. If nurse managers are to ensure the quality of patient care, the wellness of the nurses working in their units must receive attention, and therefore the success of a WWP must be measured so that adaptations can be made if necessary.

The logic model guided the components underlying the evaluation framework by illustrating how it was expected to achieve the intended outcomes. It furthermore explained the causal links that connected the programme and evaluation activities to the expected outcomes ${ }^{[17]}$, clearly defining and describing the theory of change within the framework.

The theory of change supporting a WWP was thus described and the logic model applied in the evaluation framework. It helped in planning and managing activities in the programme where evaluation was important ${ }^{[16,17]}$.

The evaluation framework comprised of the following features or components: inputs, activities, outputs and outcomes as suggested in the theory of change logic model. Additional components were context (also termed external contextual influences) and implementation factors. Connections or links indicated how the components or elements within the framework related to one another and how the theory of change would apply ${ }^{[16,20]}$.

The framework is represented in the form of a diagram, with columns and rows of boxes containing text and causal connections ${ }^{[21]}$ to conform to the components of a logic model. The framework thus comprised seven columns or sections, each representing a component. Each column consisted of a row of boxes containing text briefly indicating which subsection of a component was being dealt with.

The component Inputs described the commencement of a certain area of a programme ${ }^{[16,20]}$ such as funding, equipment, facilities, managerial leadership, the research base, tangibles, service suppliers, human resources, monitoring and evaluation feedback, as well as information about the needs of nurses.

The component Activities explained the treatments or actions undertaken to arrive at the desired programme goals or outcomes. They represented well planned and executed practical activities ${ }^{[16,20]}$ carried out within a specific time, such as the appointment of an HIV/AIDS management task team.

The component Outputs indicated the immediate results of an action or activity. Outputs reflected the amount of work that was done during programme implementation. They were expressed numerically and focussed on ways of measuring participation in a programme. Outputs furthermore pointed towards the progress of the theory of change within the framework $^{[20]}$. For example, a peer and counsellor HIV/AIDS training programme (activity) was linked to the number of persons trained (output).

The component Outcomes represented the change or benefits that occurred, indicating that the programme goals and objectives resulting from the activities and outputs had been attained. The theory of change would be accurate if the desired outcomes, such as change in attitudes and behaviours or increased knowledge, were achieved. The outcomes had a time dimension, and were identified as either short-term, medium-term or long-term outcome(s) ${ }^{[16,21]}$. Short-term outcomes were changes or benefits most closely associated with the programme outputs, such as compliance with the 
HIV/AIDS workplace strategy, goals and legislation. Implementation of a sustainable and effective WWP was an example of a medium-term outcome explaining the changes that resulted from the short-term outcomes. Long-term outcomes were changes or benefits that stemmed from the medium-term outcomes, such as the maintenance of a sustainable and effective WWP. It was furthermore important to indicate what changes were expected within which timeframe e.g whether a specific outcome should be reached with 6 or 12 months.

An additional component was included in the evaluation framework, namely Context ${ }^{[16]}$, also termed external contextual influences ${ }^{[21]}$ on a programme. The context explained the environment in which the programme would function, as well as the factors and influences that had to be taken into consideration should a programme be initiated. In the evaluation framework, context therefore comprised

- The internal and external factors that influenced the wellbeing of a nurse practitioner contending with HIV/AIDS in the workplace;

- The managerial, as well as internal and external workplace factors that had an influence on the development of a WWP.

The contextual factors could have either a positive or a negative influence on the design and delivery of the programme ${ }^{[21]}$, because these factors were not under the control of the programme.

Implementation objectives were a further component included in the evaluation framework. Their aim was to focus attention on each activity that was required to produce an output. The implementation objectives described what was being done in order to achieve the intended outcomes of the programme ${ }^{[20]}$. Ways to state implementation objectives included "to provide", "to do", and "to give".

Each column with its subsections was linked to the others by means of unidirectional arrows or simple lines and brackets. The connections indicated by a unidirectional arrow showed the direction in which the changes were expected to occur after or as a result of an activity. The connections indicated as a simple line or bracket explained which items belonged together as part of the same component ${ }^{[16]}$.

An example of an expected result of an action or activity that could be achieved after implementing a different HIV/AIDS programme was illustrated to complement the evaluation framework illustrated in Figure 1. The process of change could be interrupted and evaluated during any stage of the framework. This monitoring and evaluation process could afford the nurse healthcare manager a timely opportunity to alter a WWP ${ }^{[17]}$. An expected outcome could therefore be evaluated during any stage of the flow of events.

The nurse healthcare manager could identify the nurses' need for HIV/AIDS programmes under Inputs. Different programmes identified after conducting an impact study in the workplace would be listed under Activities. The Implement objective would then be explained as being to support and promote the health and wellbeing of the nurse practitioner. The unidirectional arrows clearly indicated the "movement" or change predicted to occur in the sequence of events. The nurse healthcare manager would utilise the feedback results after the commencement of an activity, such as VCCT visits, to indicate whether progress had been made towards achieving change under Outputs.

The change or benefits that occurred would be evaluated and classified in terms of the timeframe in which the goals or objectives that resulted from the activities and outputs were attained. Therefore, for example, the nurse healthcare manager would indicate that the first change to occur after a number of nurses made use of the VCCT programme was increased knowledge. If the unidirectional arrow indicated that the medium-term outcomes had been achieved, this would be an indication that the needs of the nurse practitioner were satisfied. The theory of change would be accurate if the long-term outcomes indicated that the nurse practitioner was able to maintain positive health and wellbeing. 


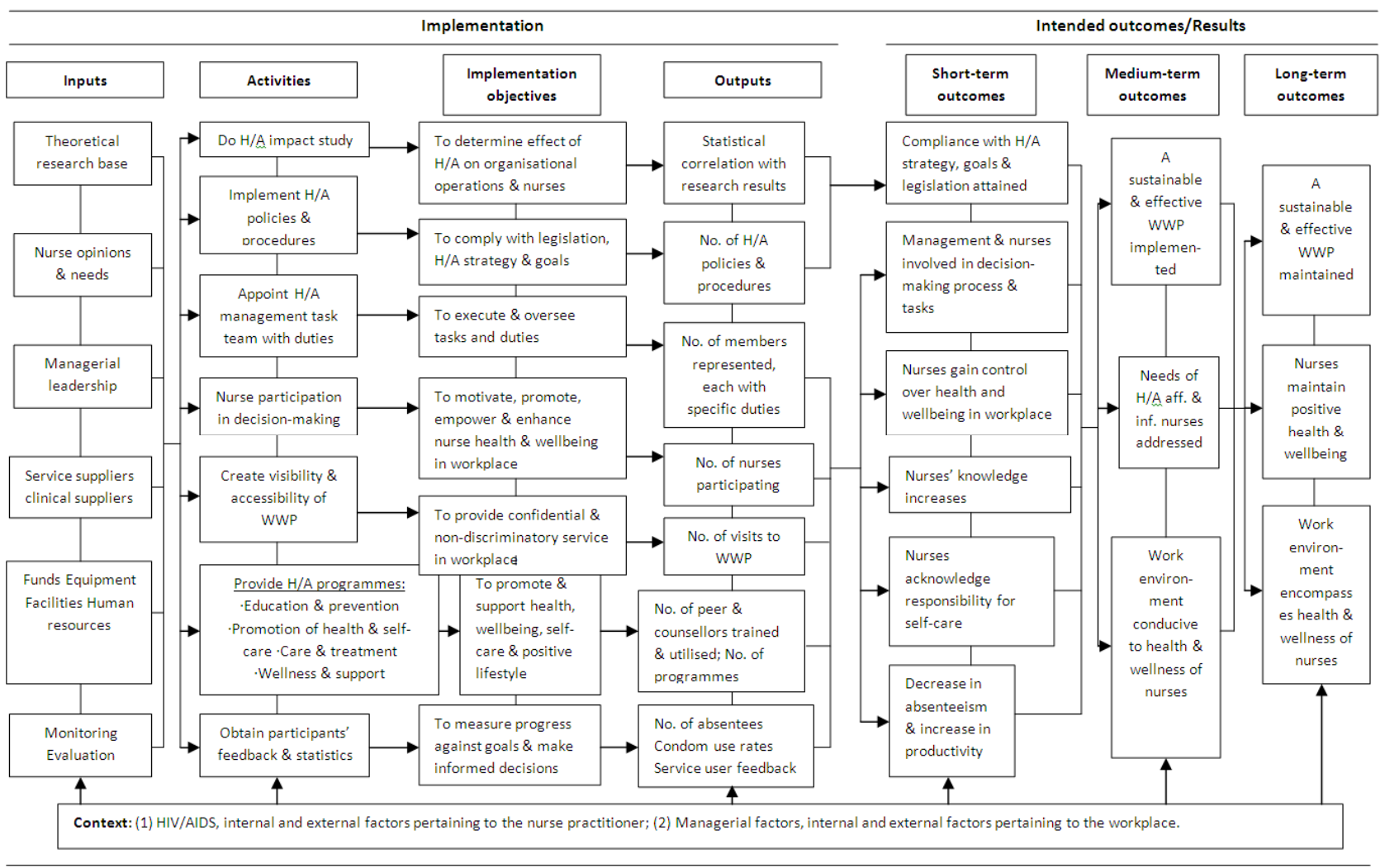

Figure 1. Logic model for an evaluation framework

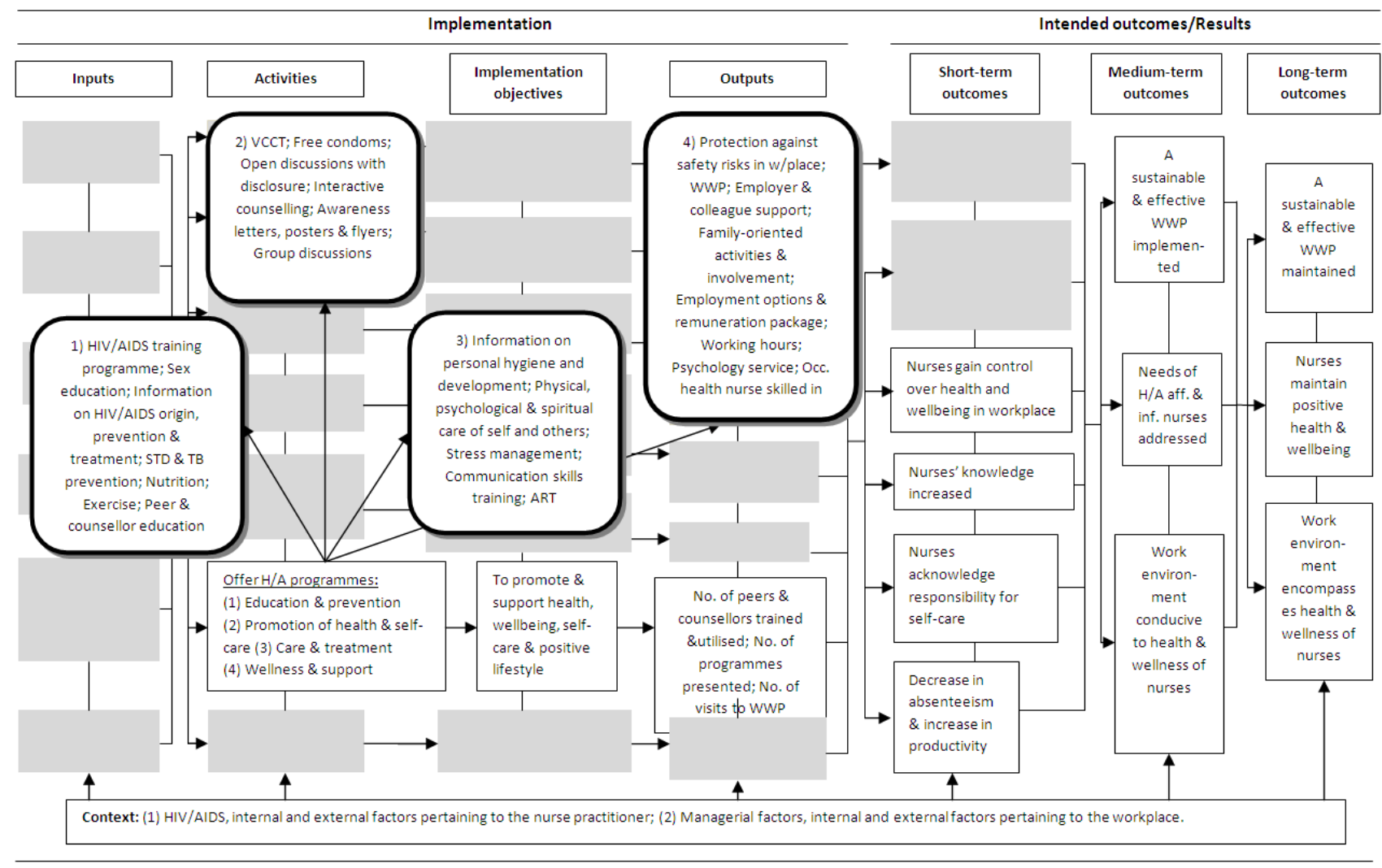

Figure 2. Logic model for an evaluation framework reflecting HIV/AIDS programme content 
A nurse healthcare manager could interrupt the flow of events at any stage to evaluate the appropriateness and effectiveness of the inputs, activities and outputs. This would enable him or her to measure the progress within a WWP against the stated goal and objectives of an organisation. It would assist in making informed decisions on HIV activities after the evaluation results had been reviewed.

Evaluation results could be obtained from statistical reports such as employee absenteeism rates and occupational accidents with blood and bodily fluids. Feedback evaluation reports regarding service users, condom use rates, requests for VCCT service and number of peer educators and counsellors in an organisation or hospital could be examined. Staff morale, general awareness and attitude towards HIV surveys, as well as satisfaction with organisational health promotion programmes and activities surveys, could be included in the evaluation strategy.

\section{Limitations}

The data-gathering was done at one large private healthcare facility where the majority of nurses were white and female; the data can therefore not be generalised. However, the data were gathered to support the development of the evaluation framework, which was informed by a thorough literature analysis.

\section{Conclusion and implications for nursing management}

Evaluation strategies are important for assessing the extent to which a WWP for nurses succeeds in attaining its expected outcomes. The evaluation framework can be utilised in different settings and environments by nurse healthcare managers to support an effective and sustainable programme, but can also be used as guide to adapt and revise the WWP to enhance the wellness of nurses, and thus ultimately the quality of patient care.

\section{References}

[1] O’Donnell MP. Health promotion in the workplace. 3rd ed. Albany: Delmar Thomson Learning. 2002: 544-571.

[2] Hartwig K, Pashman J, Cherlin E, Callaway M, Czaplinski C, Wood W, et.al. Hospital management in the context of health sector reform: a planning model in Ethiopia. International Journal of Health Planning and Management. 2008; 23(3): $203-218$. PMid:18157912 http://dx.doi.org/10.1002/hpm.915

[3] Nel PS, Swanepoel BJ, Kirsten M, Erasmus BJ, Tsabadi MJ. South African employment relations: theory and practice. 5th ed. Pretoria: Van Schaik. 2005: 326-296. PMid:16186997

[4] WORLD HEALTH ORGANIZATION. Global HIV/AIDS response. Available from: http://who.int/hiv/en/ (19 July 2012).

[5] Zuberi T, Sibanda A, Udjo E. The demography of South Africa. Armonk: Sharpe. 2005: 88-91.

[6] Van Dyk A. HIV/AIDS care \& counselling: a multidisciplinary approach. 4th ed. Pineland: Pearson Education. $2008:$ 4-469.

[7] Page J, Louw M, Pakkiri D. Working with HIV/AIDS. Cape Town: Juta. 2006: 104-112.

[8] CHAMP, D. 2006. Nurses need support. Nursing Update. 2006; 30(2): 50.

[9] Lastowka L. 8 ways to promote wellness in the workplace. Available from: www.inc.com/guides/2011/01/8-ways-to-improve-wellness-in-the-workplace.html (17 July 2012).

[10] Vass J, Phakathi S. Managing HIV in the workplace - learning from SMEs. HSRC Research Monograph. Cape Town: HSRC Press. 2006: 1-98.

[11] IFC (INTERNATIONAL FINANCE CORPORATION). Code of Practice on HIV/AIDS and the World of Work. 2002. Available from: http://www.ifc.org (15 March 2013).

[12] Thomas EP, Colvin M, Rosen SB, Zuccarini C. HIV prevalence study for the development of a HIV workplace strategy for Buffalo City Municipality. Available from: http://www.cadre.org.za/files/Buffalo_City_Report.pdf (5 June 2009).

[13] Janse van Rensburg MNS. The impact of an HIV/AIDS workplace wellness programme in a large packaging factory. Available from: http://witsetd.wits.ac.za (5 June 2009).

[14] Lunney M. The need for international nursing diagnosis research and a theoretical framework. International Journal of Nursing Terminologies and Classifications. 2008; 19(1): 28-34. PMid:18331482 http://dx.doi.org/10.1111/j.1744-618X.2007.00076.x 
[15] Doran DM, Sidani S. Outcomes-focussed knowledge translation: a framework for knowledge translation and patients outcomes improvement. Worldviews on Evidence-Based Nursing. 2007: 3-13. PMid:17355405 http://dx.doi.org/10.1111/j.1741-6787.2007.00073.x

[16] Frechtling JA. Logic modeling methods in program evaluation. San Francisco: Jossey-Bass. 2007: 5-32

[17] W.K. KELLOGG FOUNDATION. Logic model development guide. Battle Creek, Michigan. 2002: 1-57.

[18] Sandelowski M, Barroso J, Voils CI. Using qualitative meta summary to synthesize qualitative and quantitative descriptive findings. Research in Nursing \& Health. 2007; 30: 99-111. PMid:17243111 http://dx.doi.org/10.1002/nur.20176

[19] Lavoie-Tremblay M, Wright D, Desforges N, Gélinas C, Marchionni C, Drevniok U. Creating a healthy workplace for newgeneration nurses. Journal of Nursing Scholarship. 2008; 40(3): 290-297. PMid:18840214 http://dx.doi.org/10.1111/j.1547-5069.2008.00240.x

[20] McDavid JC, Hawthorn LRL. Program evaluation and performance measurement: an introduction to practice. USA: Sage. 2006: 15-54.

[21] Wholey JS, Hatry HP, Newcomer KE. Handbook of practical program evaluation. 2nd ed. San Francisco: Jossey-Bass. $2004:$ 1-25. 